\title{
High-order methods for the simulation of hydromagnetic instabilities in core-collapse supernovae
}

\author{
T. Rembiasz ${ }^{* 1}$, M. Obergaulinger ${ }^{1}$, M. Angel Aloy ${ }^{2}$, P. Cerdá-Durán ${ }^{1}$ \\ and E. Müller ${ }^{1}$ \\ ${ }^{1}$ Max-Planck-Institut für Astrophysik, 85748 Garching, Germany \\ *email: rembiasz@mpa-garching.mpg.de \\ ${ }^{2}$ Universitat de València, 46100 Burjassot (Valencia), Spain
}

\begin{abstract}
We present an assessment of the accuracy of a recently developed MHD code used to study hydromagnetic flows in supernovae and related events. The code, based on the constrained transport formulation, incorporates unprecedented ultra-high-order methods (up to 9th order) for the reconstruction and the most accurate approximate Riemann solvers. We estimate the numerical resistivity of these schemes in tearing instability simulations.
\end{abstract}

Keywords. reconstruction schemes, Riemann solvers, hydromagnetic instabilities

\section{Introduction}

If the weak magnetic fields of progenitor stars are amplified efficiently in the postcollapse phase, they may affect the dynamics of core-collapse supernovae significantly. Prime candidates for this amplification are hydromagnetic instabilities in the stellar core, e.g., magneto-convection and the magneto-rotational instability (e.g., Obergaulinger et al. 2009). Leading to small-scale turbulence, these instabilities demand the use of highly accurate methods in supernova simulations, while the wide range of physics involved and the presence of supersonic flows and strong shock waves call for flexibility and stability.

The properties of turbulence developing due to MHD instabilities can depend crucially on transport coefficients such as the resistivity, $\eta$. In any numerical simulation, numerical diffusion and dissipation, described approximately by a numerical resistivity, $\eta_{\text {num }}$, add to the effects of the physical (microscopic) resistivity. A clear distinction between both effects is important for a correct interpretation of the simulations.

Therefore, we try to quantify the numerical resistivity of our MHD code, an implementation of a finite-volume constraint-transport scheme. Here we show a comparison of the numerical resistivity for different resolutions, Riemann solvers (Lax-Friedrichs (LF) (e.g., Toro 2009), Harten, Lax, van Leer (HLL) (e.g., Toro 2009), the approximate six-stage MHD solver (HLLD) (Miyoshi \& Kusano 2005)) and reconstruction schemes (piecewise linear (PL) (e.g., Toro 2009), monotonicity preserving (MP) (Suresh \& Huynh 1997) of 5 th, 7 th and 9 th order $)$.

\section{Simulations}

We performed 2D simulations of the tearing instability in a current sheet in force-free magneto-hydrostatic equilibrium with constant gas pressure and density using similar 

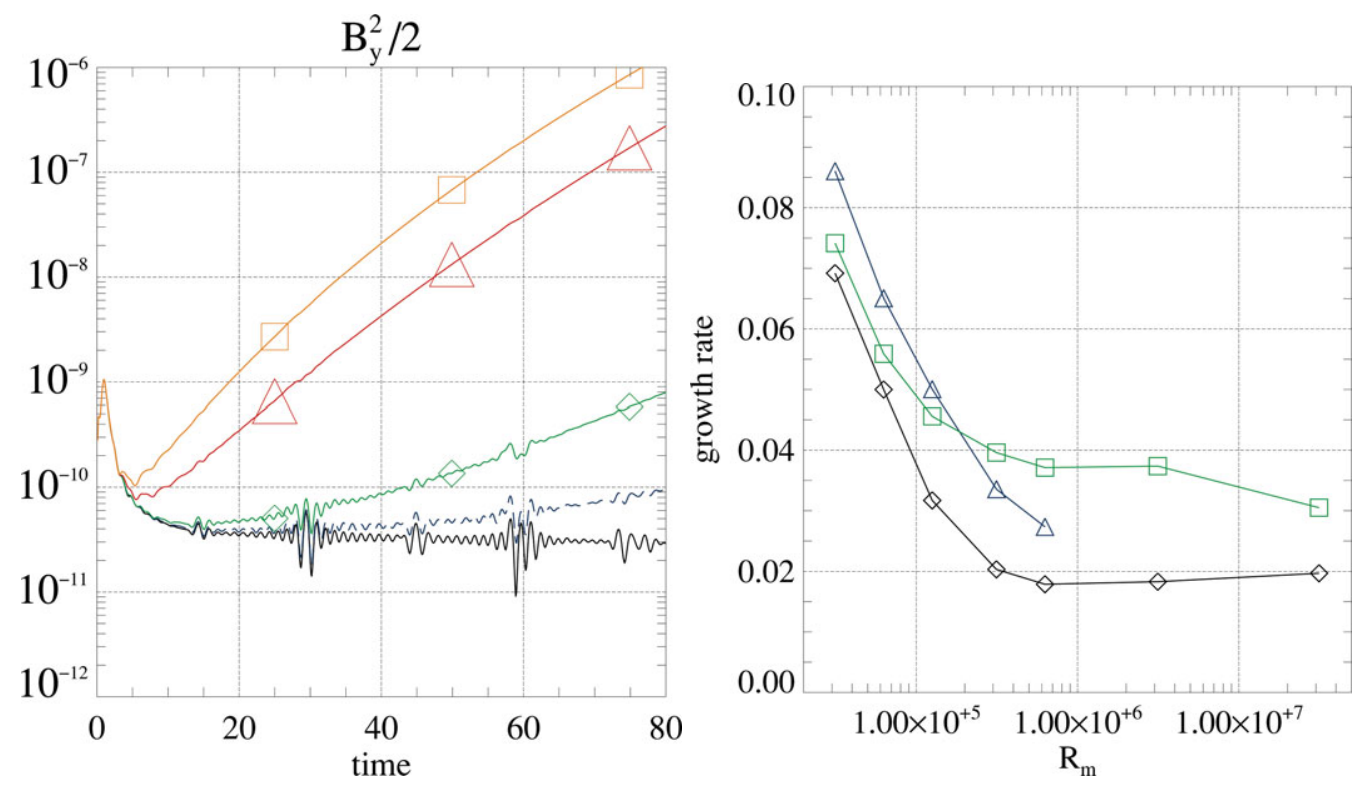

Figure 1. Left: Time evolution of $B_{y}^{2} / 2$ for different magnetic Reynolds numbers $R_{m}$. HLL Riemann solver, MP 5th order and grid of $512 \times 1024$ zones were used. $R_{m}=\pi \times 10^{4}$ (orange squares), $R_{m}=\pi \times 10^{5}$ (red triangles), $R_{m}=2 \pi \times 10^{5}$ (green diamonds), $R_{m}=2 \pi \times 10^{7}$ (dashed blue), $R_{m}=\pi \times 10^{7}$ (solid black).

Right: Growth rates for different Riemann solvers: LF (green squares), HLL (black diamonds), HLLD (blue triangles). The simulations were performed with 7 th order MP reconstruction on grid of $128 \times 256$ zones.

physical parameters as in Landi et al. (2008) for different resistivities. The initial configuration is perturbed with transverse velocity fluctuations, which are much smaller than both the Alfvén and the sound speed. Due to non-zero resistivity the tearing instability sets in. This can bee seen in Fig. 1, depicting the time evolution of the average of the transverse $(y)$ component of the magnetic energy. It grows exponentially during the first phase of evolution and its growth rate is proportional to resistivity. (Note that the onset of the instability is delayed for higher Reynolds numbers, defined as $R_{m}=c_{A} / L_{y} \eta$, where $L_{y}$ is the transverse box size.) However, this proportionality holds only above a critical resistivity $\eta^{*}$ being approximately equal to the numerical resistivity $\eta_{\text {num }}$. This sets the maximum Reynolds number $R_{m}^{*}$ which can be resolved (for a given scheme and resolution).

Resolution studies are presented in the left panel of Fig. 2. With the coarsest grid we are not able to resolve Reynolds numbers higher than $R_{m}^{*} \approx 10^{5}$, because the numerical resistivity dominates over the physical one. Doubling the number of grid zones reduces the numerical resistivity approximately fourfold for the used scheme. In the right panel of Fig. 2 we compare different reconstruction methods. All simulations were performed with a fixed number of the grid zones. 9th order MP is approximately twice less resistive than 7th order MP and four times less resistive than 5th order MP. The PL scheme seems to be less reliable than MP schemes for these simulations. A comparison of three different Riemann solvers is shown in the right panel of Fig. 1. 

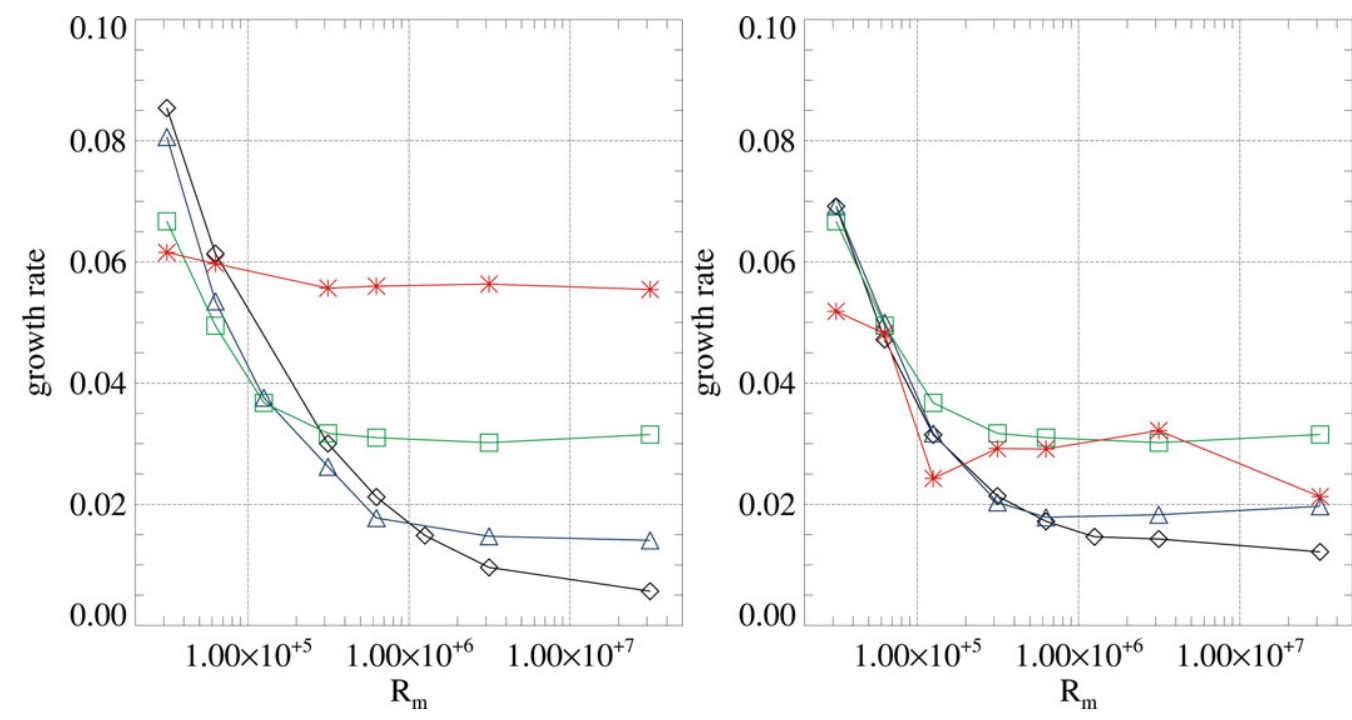

Figure 2. Left: Resolution studies with HLL Riemann solver and MP of 5th order. Grids of: $64 \times 128$ (red asterisks), $128 \times 256$ (green squares), $256 \times 512$ (blue triangles), $512 \times 1024$ zones (black diamonds) were used.

Right: Growth rates for different reconstruction schemes: PL with a combination of minmod and MC slope limiter (red asterisks), MP of 5th order (green squares), MP of 7 th order (blue triangles), MP of 9th order (black diamonds). In the simulations HLL Riemann solver and grid of $128 \times 256$ zones were used.

\section{Conclusions}

We conclude that with the aforementioned simulations we are able to measure the numerical resistivity of our code. This allows us to choose a proper grid resolution and reconstruction schemes such that the numerical resistivity is lower than the microscopic resistivity with which we want to simulate the physical phenomena of interest.

In the performed tests we reduced by approximately the same factor the numerical resistivity of the simulations with 5 th order MP, either by choosing 9 th order MP, or by doubling the resolution. The latter is computationally much more expensive. Therefore, in order to resolve higher Reynolds numbers, one should start by choosing higher order reconstruction methods, rather than by increasing resolution, if the computational time is a matter of concern.

\section{References}

S. Landi, P. Londrillo, M. Velli, \& L. Bettarini Physics of Plasmas 15 (2008) 012302

T. Miyoshi \& K. Kusano, J. Comput. Phys. 208 (2005) 315-344

M. Obergaulinger, P. Cerdá-Durán, M. Angel Aloy, \& E. Müller, A\&A 1323 (2009)

A. Suresh \& H. T. Huynh, J. Comput. Phys. 136 (1997) 83

E. Toro, Riemann Solvers and Numerical Methods for Fluid Dynamics, 3rd edition, SpringerVerlag Berling Heidelberg 2009 